\title{
Clasificación de variables morfológicas del duraznero (Prunus persica L. Batsch) 'Jarillo' en la montaña santandereana Colombiana mediante análisis discriminante lineal
}

\section{Classification with linear discriminant analysis of morphological variables in peach (Prunus persica L. Batsch) 'Jarillo' in the Santandereana Mountains, Colombia}
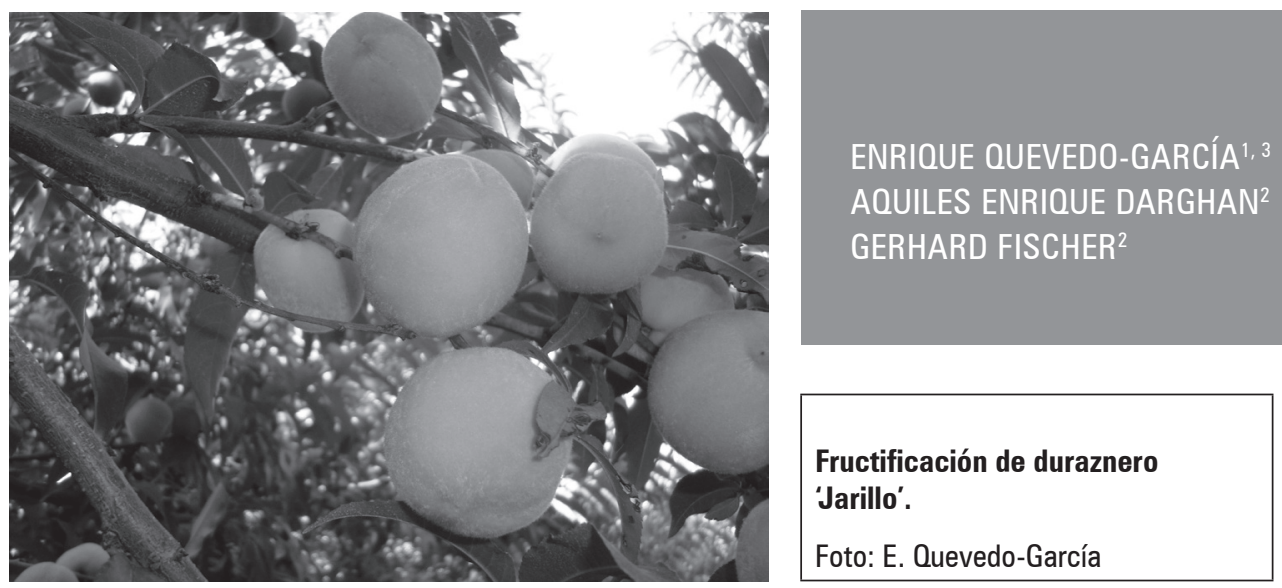

\section{RESUMEN}

Durante el 2014 en la provincia de Pamplona, Colombia, se realizó un estudio sobre la variedad de duraznero Jarillo, con el fin de clasificar una serie de variables morfométricas distribuidos en tres fincas de diferenter altitud (Pamplona, $2.170 \mathrm{msnm}$; Chitagá, $1.870 \mathrm{msnm}$ y Pamplonita, $1.670 \mathrm{msnm}$ ). Para el muestreo de árboles se utilizó el hipercubo latino condicional. En las hojas se midió el ancho por la longitud de limbos y el área del limbo usando el software ImageJ. En los frutos se midió el diámetro longitudinal sobre ecuatorial y los pesos secos. Se aplicó la técnica del análisis discriminante lineal, el cual se complementó con el diagrama de cajas por finca con los valores de la función discriminante para las muestras de diferentes grupos y se finalizó con la gráfica de dispersión de las dos mejores funciones discriminantes. Esta clasificación sugiere un manejo agronómico diferenciado entre Pamplonita y Chitagá, sobre todo en la cosecha, pues esta involucra el peso seco de los frutos. Existe un área común entre Pamplona y Pamplonita, lo cual puede atribuirse a que ambas se encuentran en la cuenca del Catatumbo. Los valores de largo por ancho del limbo y área del limbo fueron caracteres fuertemente discriminantes en este estudio, son susceptibles de variar debido a factores ambientales. El estudio concluyó que las fincas Chitagá y Pamplonita se discriminan claramente en lo que respecta a las variables medidas, alcanzando la función discriminante un 89,3\% de separación en las variables.

Facultad de Ciencias Agrarias, Departamento de Agronomía, Universidad de Pamplona. Pamplona (Colombia).

Facultad de Ciencias Agrarias, Departamento de Agronomía, Universidad Nacional de Colombia. Bogotá (Colombia).

Autor para correspondencia.enriquegarcia@unipamplona.edu.co 


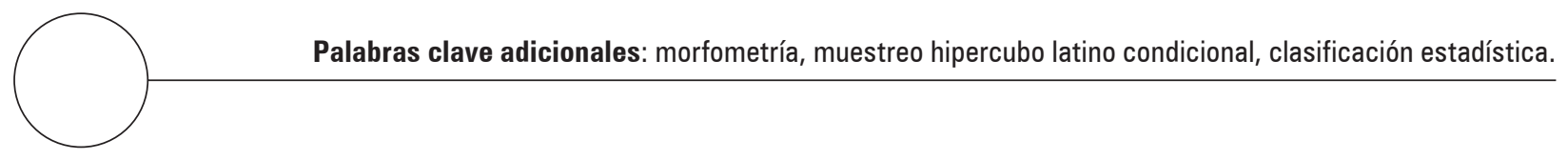

ABSTRACT

During 2014, in the province of Pamplona, Colombia, a study on the peach variety Jarillo was carried out in order to classify a series of morphometric variables in this crop, grown at three different altitudes (Pamplona, 2,170 $\mathrm{m}$ a.s.l., Chitaga, 1,870 $\mathrm{m}$ a.s.1., and Pamplonita, 1,670 $\mathrm{m}$ a.s.l). The conditional Latin hypercube sampling approach was used to select samples of the trees. In the leaves, the width was measured with the length of the limbs and the limbus area using the software ImageJ. In the fruits, the longitudinal $r$ on the equatorial diameter and dry weight were measured. The linear discriminant analysis technique was applied, which was complemented with boxes diagram by farm with the values of the discriminant function for the samples of the different groups and ended with the scatter plot of the two best discriminant functions. This classification suggested different agronomic management plans for Pamplonita and Chitagá, especially for the harvest that involved the dry weight of the fruits. There is a common area between Pamplona and Pamplonita, which can be attributed to the fact that both are in the Catatumbo basin. The values of length and width of the limbus and limbus area were highly discriminating characteristics in this study and were susceptible to change because of environmental factors. The study concluded that the farms Chitagá and Pamplonita were clearly discriminated according to the measured variables, reaching discriminant function with $89.3 \%$ separation in the variables.

Additional key words: morphometric, conditional Latin hypercube sampling, statistical classification.

Fecha de recepción: 09-01-2017 Aprobado para publicación: 15-04-2017
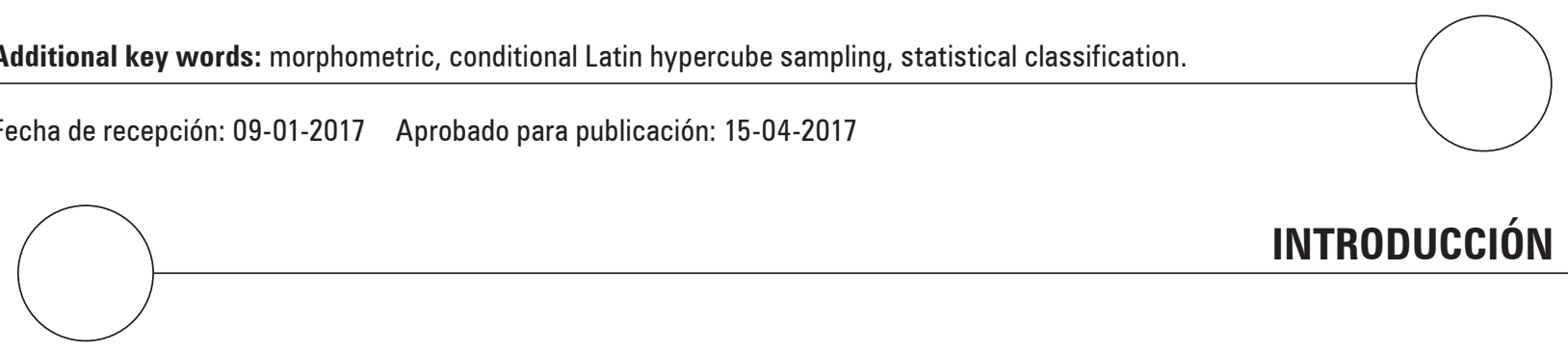

INTRODUCCIÓN

Los durazneros (Prunus persica L. Batsch), como otros caducifolios, encuentran condiciones favorables para su crecimiento en varias regiones tropicales, para producir anualmente una o más cosechas (Fischer et al., 2010). Es decir, según su nombre científico, sería originario de Persia (Irán), mientras escrituras chinas del 2.000 a.C. indican que es procedente de la China (Cárdenas y Fischer, 2013).

La producción agronómica de durazno en Colombia se concentra en los departamentos andinos de Boyacá, Cundinamarca, Norte de Santander y Santander (MADR, 2012), en municipios ubicados entre los 1.600 y 3.332 msnm (Carranza y Miranda, 2013). En la provincia de Pamplona se encuentra un área sembrada de 830 ha de duraznero, distribuidas de la siguiente forma: en el municipio de Pamplonita 82,3 ha, en Pamplona 43,6 ha, en Chitagá 236,5 ha, en Cácota 210,5 ha y en el municipio de Silos 170,8 ha (Fernández, 2013). La variedad Jarillo de bajo requerimiento de frío es de origen venezolano y es sembrada en el departamento de Norte de Santander (Campos, 2013).
El estudio de la morfometría en las ciencias agrícolas juega un importante papel no solo desde el punto de vista de la sistemática de las plantas (Henderson, 2006) sino en el desarrollo de estrategias de manejo específicas en los cultivos gracias a la posibilidad de generar clasificadores, tal como las funciones discriminantes. Cuando se tienen el conjunto de variables morfométricas por finca (Chitagá a $1.870 \mathrm{msnm}$, Pamplona a $2.170 \mathrm{msnm}$ y Pamplonita a $1.670 \mathrm{msnm}$ ), resulta de interés estudiar si realmente las fincas discriminan las variables involucradas, en este sentido, el análisis discriminante lineal (LDA) se trata de una técnica de análisis multivariante que es capaz de aprovechar las relaciones existentes entre una gran cantidad de variables independientes para maximizar la capacidad de discriminar, es un método útil para el reconocimiento de patrones, donde se busca una combinación lineal de funciones que caractericen o separen dos o más clases de objetos o eventos, que en este caso, se asocian a las fincas. La combinación resultante puede ser utilizada como un clasificador lineal, que es un tipo particular de clasificador, que calcula la decisión por combinación lineal de las muestras. Este término 
puede ser reemplazado por discriminación lineal, o superficie de separación lineal. Para los estadísticos, estos métodos a veces se clasifican como métodos de análisis discriminante o, más comúnmente, para la reducción de dimensionalidad antes de una clasificación posterior (Jobson, 1992).

En el caso general, si se tienen $m$ muestras aleatorias de diferentes grupos, con tamaños $n_{1}, n_{2}, \ldots n m$, con valores disponibles para $p$ variables $\mathrm{X}_{1}, \mathrm{X}_{2}, \ldots, \mathrm{X}_{\mathrm{p}}$ para cada miembro de la muestra, de este modo, los datos para un discriminante conforman una matriz de dimensión $m \times p$. Los datos para un análisis discriminante no necesitan ser estandarizados para tener media cero y varianza 1, como es habitual con otras técnicas. Esto se debe a que la función discriminante no se ve afectado de manera importante por la escala de las variables individuales (Manly y Navarro, 2017). Como en otros análisis, resulta importante verificar si se tienen algunas observaciones que se comportan de manera diferente a la mayoría de los datos, o por la presencia de observaciones discordantes, valores atípicos o influyentes (Beckman y Cook, 1983).

Este tipo de análisis se puede usar en la práctica para caracterizar la morfométrica del fruto de mango de varias localidades productoras en Venezuela, se escogieron variables discriminantes tales como la masa total del fruto, del epicarpio, de la semilla y del mesocarpio, el diámetro ecuatorial y polar, las proporciones $(\%)$ de las diferentes partes del fruto, humedad del fruto, fibra dietética, cenizas, minerales $\mathrm{Ca}, \mathrm{Fe}$, $\mathrm{Na}, \mathrm{K}, \mathrm{Mg}$ y Zn, sólidos solubles totales, acidez total titulable, pH, ácido ascórbico y actividad de agua; la diferencia se estableció para las zonas productoras por medio del análisis discriminante lineal (Garrido et al., 2013).

Para realizar el análisis discriminante, se pueden usar también variables asociadas a la hoja; se han considerado la peciolo, longitud de la hoja y ancho de la hoja en Minthostachys teniendo en cuenta dos centros de origen (Gómez et al., 2013). A través de un análisis discriminante, usando como variables discriminantes el largo total y el ancho máximo de la hoja, la distancia entre lóbulos, la longitud de los lóbulos a distintos niveles y el número de venas, se encontró una tendencia en las muestras de Montrichardia arborescens (L.) Schott (Araceae), provenientes del delta Amacuro en Venezuela que se diferencian de las hojas del resto de las regiones estudiadas, por tener mayor longitud del lóbulo y menor largo total de la hoja (Gordon-Colón et al., 2013). Estos autores indican que esta especie presenta una alta variabilidad foliar, encontrándose variaciones regionales en la forma foliar, el rasgo funcional más común en plantas acuáticas. En Bromus valdivianus Phil. se caracterizaron 125 accesiones de esta especie, colectadas en diferentes áreas en el sur de Chile para analizar su variabilidad fenotípica. Un total de 34 variables discriminantes fueron sometidas a análisis de componentes principales, agrupamiento y análisis discriminante. Los dos primeros componentes principales explicaron el $51 \%$ de la variación total. En el análisis de agrupamiento se distinguieron seis grupos, dos grupos contrastantes, uno formado por accesiones con características asociadas a una buena producción de forraje y otro asociado a características de mayor producción de semillas. Estos resultados fueron útiles en la selección de material promisorio de utilización inmediata (Blanco et al., 2010).

El objetivo de este estudio fue la identificación de las variables morfométricas de la hoja y fruto que mejor discriminaron entre los pisos térmicos para ayudar a su clasificación y la evaluación del poder discriminante de cada una de ellas.

\section{MATERIALES Y MÉTODOS}

\section{Material vegetal y caracterización de la zona de estudio}

Se usó la variedad de duraznero Jarillo, importado de Venezuela, cuyos frutos presentan características como piel y pulpa amarilla, tamaño pequeño, forma redondeada de producción prolífica pero ligeramente más tardía que la de 'Gran Jarillo'. Asimismo la variedad se ha establecido con buena adaptación en el departamento de Norte de Santander en los municipios de Pamplona, Chitagá, Silos y Cácota. Las plantas son de gran tamaño debido a la distancia de plantación establecida de 6 a $7 \mathrm{~m}$ entre plantas y surcos (Campos, 2013), distribuidos en condiciones homogéneas de pendiente y manejo agronómico que conformó la parcela de estudio de árboles de duraznero en plena producción agronómica y con una edad mayor a los 10 años donde se estabiliza la producción (Avilán et al., 2009).

La investigación se llevó a cabo en tres fincas ubicadas en zonas geográficas y altitudinales diferentes en el Norte de Santander: 
1) Pamplona, finca "Las Delicias", vereda Chíchira, con coordenadas $70^{\circ} 22^{\prime} 43,6^{\prime \prime} \mathrm{N}$ y $72^{\circ} 37^{\prime} 41,1^{\prime \prime} \mathrm{W}$, perteneciente a la Cuenca del Catatumbo; con una altitud de $2.170 \mathrm{msnm}$, temperatura media $16^{\circ} \mathrm{C}$ y una precipitación de $933,9 \mathrm{~mm}$ año-1 en régimen bimodal.

2) Chitagá, finca "El Recuerdo", vereda Carrillo, con coordenadas $70^{\circ} 11^{\prime} 15^{\prime \prime} \mathrm{N}$ y $72^{\circ} 39^{\prime} 7,3^{\prime \prime} \mathrm{W}$, perteneciente a la cuenca del Orinoco; con una altitud de 1.870 msnm, temperatura media de $18^{\circ} \mathrm{C}$ y una precipitación de $879,5 \mathrm{~mm}$ año-1 en régimen unimodal.

4) Pamplonita, finca "Bella vista", vereda Bataga, con coordenadas $70^{\circ} 26^{\prime} 18,1^{\prime \prime} \mathrm{N}$ y $72^{\circ} 38^{\prime} 9^{\prime \prime} \mathrm{W}$, perteneciente a la cuenca del Catatumbo; con una altitud de $1.670 \mathrm{msnm}$, temperatura media $20^{\circ} \mathrm{C}$ y con precipitaciones frecuentes y acumuladas anuales que superan los $1.200 \mathrm{~mm}$ en régimen bimodal.

\section{Variables morfométricas y pesos secos}

En el estudio se involucró una matriz de datos en la fase fenológica de cosecha en la variedad jarillo, para el cual se dispuso de cuatro variables, a saber, el producto del largo por ancho $\left(\mathrm{cm}^{2}\right)$, área foliar $\left(\mathrm{cm}^{2}\right)$, cociente entre el diámetro ecuatorial y longitudinal de los frutos y el peso seco asociado(gramos). El área del limbo fue determinada en 240 hojas maduras con 2,5 meses de edad aproximadamente por las tres fincas (80 por finca), las cuales fueron muestreadas en el estado fenológico de cosecha, mediante el uso del programa de dominio público ImageJ el cual realiza análisis de imágenes (Rasband, 2010). El proceso empezó con la descarga de la foto (extensión jpg) en el programa y la inclusión del largo del limbo $(\mathrm{cm})$ como parámetro de entrada, el cual se midió previamente con un pie de rey electrónico marca Ubermann (Fa. Sodimac, Santiago, Chile). Con estos parámetros el software estimó el área del limbo $\left(\mathrm{cm}^{2}\right)$. Además se midió el ancho (eje mayor en $\mathrm{cm}$ ) y largo de los limbos $(\mathrm{cm})$ y se obtuvo su producto $\left(\mathrm{cm}^{2}\right)$, por otro lado, se calculó el cociente del diámetro ecuatorial del fruto $(\mathrm{cm})$ y el diámetro longitudinal del fruto $(\mathrm{cm})$. En lo que respecta al peso seco de los frutos (g), este se obtuvo después de secados los frutos, usando un horno eléctrico de secado de circulación forzada de aire caliente seco marca Mettler Toledo (Fa. Mettler Toledo, Columbus, $\mathrm{OH}$ ), secando a $60^{\circ} \mathrm{C}$ hasta peso seco constante, finalmente se registró la masa seca, obtenida mediante una balanza electrónica con aproximación de dos dígitos, de marca Lexus con rango de medida 3.600 g y precisión de 0,01 g. Estas variables conformaron una matriz de datos de 240 muestras tomadas aleatoriamente en tres fincas asociadas a tres altitudes, dos en la cuenca del Catatumbo (Pamplonita: $1.670 \mathrm{msnm}$ y Pamplona: $2.170 \mathrm{msnm}$ ) y otra en la cuenca del Orinoco (Chitagá: $1.870 \mathrm{msnm}$ ), correspondiéndose los valores extremos a la cuenca del Catatumbo.

\section{Métodos de muestreo}

En el muestreo de árboles por finca, se utilizó el hipercubo latino condicional (Minasny et al., 2012), el cual es una malla cuadrada que contiene posiciones muestrales y en la que únicamente hay una muestra en cada fila y en cada columna. No pueden existir filas o columnas con dos muestras. Un hipercubo latino es la generalización de este concepto a un número arbitrario de dimensiones, y en concordancia con ello existe, una muestra en cada hiperplano que la contenga, con la malla cuadrada se identificaron 48 árboles (6 filas y 8 columnas) involucrando como variable auxiliar la proyeccion del área foliar de cada árbol que fue aproximadamente elíptico, acercándose al área con la relación pab, donde a se asoció al semi-eje mayor (largo; m) y $b$ al semi-eje menor (ancho; $\mathrm{m}$ ). El muestreo se fijó para cada finca, y con la ayuda de la librería clhs del software R se generó una muestra de ocho árboles por piso, de los cuales se tomaron 10 hojas del tercio medio y 10 frutos del mismo tercio, a los cuales se les midió cada una de las variables antes descritas. Estas muestras conformaron una matriz de 240 observaciones discriminadas en 80 por finca (cada una ubicada en un piso altitudinal).

\section{Análisis estadístico}

En principio se construyó un diagrama de dispersión matricial exploratorio de las relaciones lineales entre múltiples variables y presencia de observaciones atípicas. Para hacer más objetiva la asociación lineal se construyó la matriz de correlaciones de Pearson entre todos los pares de variables. Para finalizar el análisis descriptivo, se obtuvo la media, la varianza y el coeficiente de variación de cada variable discriminado por piso térmico. Para iniciar el análisis inferencial, se realizó una prueba de Normalidad Multivariante (prueba de Royston) y a continuación se aplicó la técnica del análisis discriminante lineal (LDA) para encontrar las combinaciones lineales de las variables originales que dieron la mejor separación posible entre las fincas. El LDA se complementó con el diagrama de cajas 
para los valores de la función discriminante para las muestras de diferentes fincas. Finalmente se obtuvo el gráfico de dispersión de las dos mejores funciones discriminantes, las que obligaron a presentar una serie de estadísticas descriptivas por piso térmico para evidenciar la separación de los grupos.

\section{RESULTADOS Y DISCUSIÓN}

A partir del análisis de correlación, todos los pares variables analizados mostraron un $P \leq 0,01$. Todas las correlaciones resultaron altamente significativas, lo que justificó el análisis inferencial, tal como lo describió William (1983) en su conjunto de observaciones sobre el uso del análisis discriminante en ecología.

De las dos funciones discriminantes construidas, la primera expresa de mejor manera la separación de variables $(89,3 \%)$. Estas funciones se graficaron en un diagrama de puntos en el cual se visualizó la separación alcanzada por las funciones para cada uno de los pisos térmicos involucrados. El estudio concluyó que los grupos de fincas con durazneros ( $>10$ años) se discriminaron en forma clara para las fincas de Pamplonita y Chitagá, no así para la de Pamplona, en la cual se evidenció mayor solapamiento en los puntos con respecto a las dos fincas remanentes. Esta clasificación sugiere un manejo agronómico diferenciado entre Pamplonita y Chitagá, sobre todo en la cosecha, pues esta involucra el peso seco de los frutos.
La separación encontrada facilitó la construcción de medidas estadísticas descriptivas por finca para cada variable. La fuente de la separación de las fincas, proviene del producto del ancho por largo de los limbos, el cual resultó mayor en Chitagá $\left(64,125 \mathrm{~cm}^{2}\right)$ y menor en Pamplonita $\left(49,036 \mathrm{~cm}^{2}\right)$. También provino del peso seco del fruto, el cual resultó mayor en Pamplonita $(63,158$ g) y menor en Chitagá (40,655 g). Los valores de las medidas descriptivas de cada variable en su máxima y mínima (promedios) pertenecen a las fincas diferenciadas en forma clara. Al igual que Delgado et al. (2008) quien estudió variables discriminantes para discriminar localidades para la clasificación de ambientes y obtuvo valores para realizar la discriminación de los ambientes en un valor mayor de 73\%, lo que permitió sugerir manejos culturales diferentes gracias al análisis discriminante al igual que en la tres fincas analizadas en el presente trabajo.

En la figura 1 se ilustran los diagramas de dispersión matriciales exploratorios de las relaciones lineales entre las múltiples variables. Son particularmente útiles en la localización de las variables específicas que podrían tener correlaciones similares.

En la tabla 1 se observan seis pares de correlaciones, las cuales presentaron un valor de $P \leq 0,001$, lo que evidencia asociación lineal entre pares de variables, lo cual es importante para el ajuste del modelo de análisis discriminante. Las gráficas se escriben en una línea diagonal desde la parte superior izquierda a la inferior

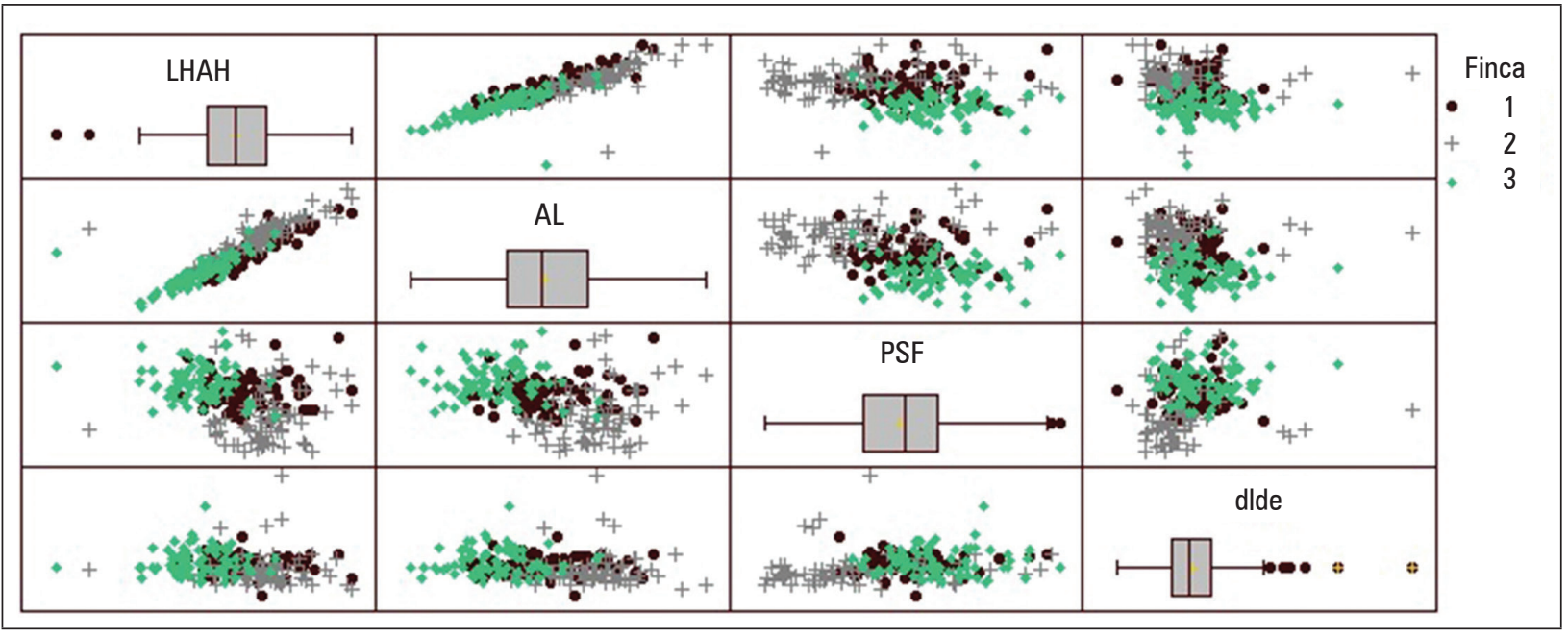

Figura 1. Diagrama de dispersión matricial. En la diagonal se describen las densidades univariantes para cada variable (LHAH: producto del largo por el ancho de hojas en $\mathrm{cm}^{2}$; AL: área del limbo en $\mathrm{cm}^{2}$; PSF: peso seco de los frutos en gramos; dlde: cociente entre diámetro longitudinal y diámetro ecuatorial). Fincas de duraznero (1) Pamplona, (2) Chitagá y (3) Pamplonita. 
derecha. Las densidades centrales muestran un comportamiento normal univariado aproximado desde el punto de vista gráfico, lo cual no garantiza normalidad multivariante, tal como lo describen Oppong y Agbedra (2016). Para obtener mayor objetividad se calculó la matriz de correlaciones de Pearson (Tab. 1).

Tabla 1. Matriz de correlaciones de Pearson. Todos los pares de correlaciones resultaron altamente significativas $(P \leq 0,001)$, LHAH: producto del largo y ancho de hojas; AL: área del limbo; dlde: cociente del diámetro longitudinal y ecuatorial, PSF: peso seco de los frutos de durazno.

\begin{tabular}{|l|c|c|c|c|}
\hline & LHAH & AL & dlde & PSF \\
\hline LHAH $\left(\mathrm{cm}^{2}\right)$ & 1,00 & 0,930 & 0,338 & $-0,303$ \\
\hline AL $\left(\mathrm{cm}^{2}\right)$ & 0,930 & 1,00 & 0,364 & $-0,344$ \\
\hline dlde & 0,338 & 0,364 & 1,00 & $-0,300$ \\
\hline PSF (g) & $-0,303$ & $-0,344$ & $-0,300$ & 1,00 \\
\hline
\end{tabular}

Con el análisis discriminante lineal se encontraron las combinaciones lineales de las variables originales (las cinco variables) que dieron la mejor separación posible entre los grupos (cultivos asociados a pisos térmicos). Previo a la construcción de las funciones discriminantes lineales, se realizó la prueba de Normalidad Multivariante (Royston), para la cual se obtuvo normalidad multivariante al 2,5\% (lo cual resulta más conveniente en sentido práctico en comparación con la transformación de los datos). Sin embargo, fueron eliminadas 10 observaciones en total, quedando para Pamplona 79 observaciones, Chitagá con 74 y para Pamplonita las 77 observaciones restantes. Al separar

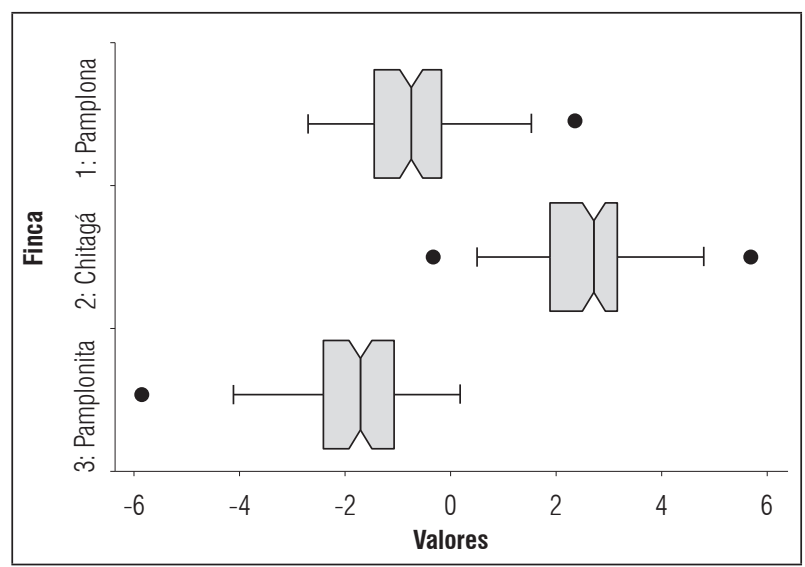

Figura 2. Diagramas de caja para la primera función discriminante para las tres fincas. las fincas se obtuvo que el número máximo de funciones discriminantes útiles fue de dos, de este modo, las dos funciones resultaron ser:

LD1: 0,0688LHAH-0,2831AL+0,0377PSF-3,1883DLDE (1)

LD2: 0,3040LHAH-0,3679AL+0,0053PSF-1,7852DLDE

La proporción de traza que asociada a cada función estimada fue de $89,3 \%$ para LD1 y $10,7 \%$ para LD2 y representan la separación porcentual obtenida por cada función discriminante, se escogio la primera función LD1 para efectos de la separación de los grupos de fincas. Además de las funciones escogidas por su mayor estimación, se construyó el diagrama de cajas de los valores de LDA con los valores de la mejor función discriminante para las muestras de diferentes fincas. El diagrama de cajas de la primera función discriminante se ilustra en la figura 2.

Aunque es clara la separación de la finca del sector de Chitagá con Pamplonita (Fig. 2), existe un área común con Pamplona, lo que implicó más similaridad entre Pamplona y Pamplonita, lo cual puede atribuirse a que ambas se encuentran en la cuenca del Catatumbo, lo que no resulta extraño, pues es posible que se den estas similaridades aún en cambios de altitud en la misma cuenca, tal como reportaron Pintaluba y Luaces (2013) en su estudio de caracterización de frutas comestibles de especies nativas en la cuenca del río Paraná en Argentina.

Una visualización de las similaridades o la discriminación entre fincas, se obtuvo mediante el gráfico de dispersión de las dos mejores funciones discriminantes, con los puntos de datos etiquetados por finca (Fig. 3).

Sin lugar a dudas, es más clara la separación que ocurre para el grupo de Chitagá con respecto a Pamplonita (signo de suma y signo de multiplicación respectivamente), mientras que el grupo de Pamplona tiene una región más marcada con Pamplonita que con Chitagá, por lo que el comportamiento de las variables en Chitagá son claramente diferentes al grupo de Pamplonita por influencia del medio ambiente diferencial sobre el crecimiento vegetativo y reproductivo (Fischer, 2013), al encontrarse en cuencas geográficas diferentes. El factor climático más importante de la altitud es la temperatura que influye la fenología a través del inicio y de la duración de los estados fenológicos y finalmente el rendimiento de la planta (Fischer et al., 2016). 

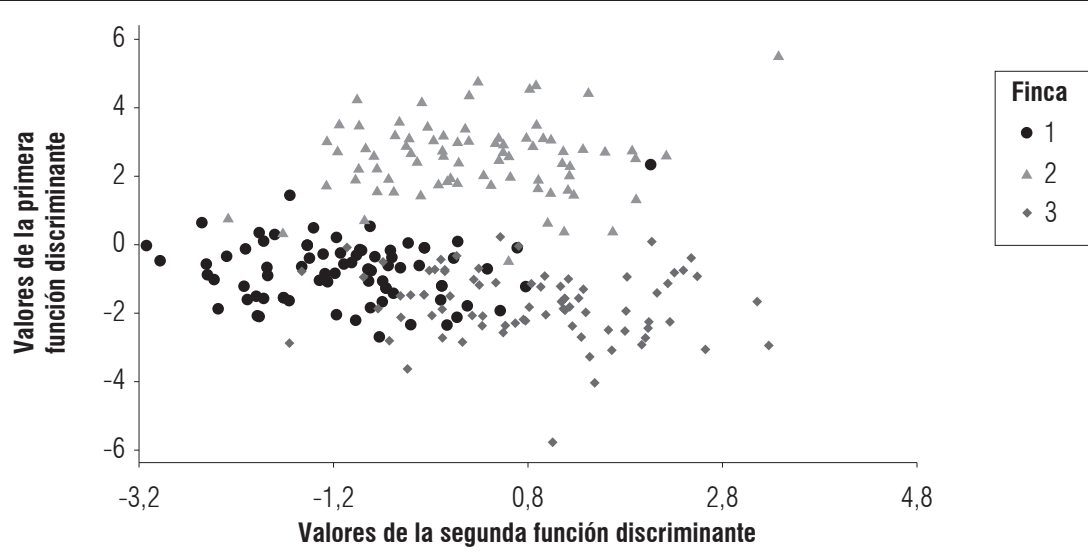

Figura 3. Diagrama de puntos para los valores de las dos primeras funciones discriminantes en las tres fincas. Fincas de duraznero (1) Pamplona, (2) Chitagá y (3) Pamplonita.

Finalmente, debido a la separación obtenida, se presentan las estadísticas descriptivas para las variables evaluadas en cada piso térmico o grupo (tabla 2).

\begin{tabular}{|c|c|c|c|}
\hline & \multicolumn{3}{|c|}{$\begin{array}{l}\text { Estadísticas descriptivas (promedio y coeficien- } \\
\text { tes de variación, en paréntesis) por finca. LHAH: } \\
\text { producto del largo y ancho de hojas; AL: área del } \\
\text { limbo; dlde: cociente entre diámetro longitudinal y } \\
\text { ecuatorial del fruto y PSF: peso seco de los frutos } \\
\text { de durazno. }\end{array}$} \\
\hline $\begin{array}{c}\text { Piso } \\
\text { Variable }\end{array}$ & $\begin{array}{l}\text { Pamplona } \\
(n=79)\end{array}$ & $\begin{array}{l}\text { Chitagá } \\
(n=74)\end{array}$ & $\begin{array}{l}\text { Pamplonita } \\
\qquad(n=77)\end{array}$ \\
\hline LHAH $\left(\mathrm{cm}^{2}\right)$ & $59.877(12,8 \%)$ & $64.125(11,1 \%)$ & $49.036(13,1 \%)$ \\
\hline $\mathrm{AL}\left(\mathrm{cm}^{2}\right)$ & 39.368 (11,9\%) & $44.922(9,7 \%)$ & $33.387(16,4 \%)$ \\
\hline dlde & $1.167(4,6 \%)$ & $1.224(4,3 \%)$ & $1.152(6,7 \%)$ \\
\hline PSF (g) & $54.792(18,4 \%)$ & $40.655(46,6 \%)$ & $63.158(19,1 \%)$ \\
\hline
\end{tabular}

Las variables LHAH y AL pertenecen a las estadísticas más altas en el grupo de Chitagá tal como lo muestra la tabla 2, lo que sugiere que los valores foliares son mayores, posiblemente por influencia de factores ambientales tales como la lluvia, la que tiene un patrón monomodal en la Cuenca del Orinoco y diferente a las otras dos fincas que presentan un comportamiento bimodal por encontrarse en la cuenca del Catatumbo. Por otro lado, aunque LHAH y AL fueron caracteres fuertemente discriminantes en este estudio, son susceptibles de variar debido a factores ambientales y en algunos casos, por causas de tipo fisiológico, asociada con la edad de la planta y su dinámica de crecimiento como se concluye para otros cultivos (Arcila y Chaves, 1995). La variación en el tamaño de las hojas podría deberse también a la intensidad y la calidad de la luz fotosintéticamente activa, a mecanismos de adaptación como la plasticidad, a factores edáficos y a las variaciones estacionales (Aribal et al., 2017; Fischer, 1995).

Por su parte, los frutos son más redondeados en Pamplonita como lo muestra la tabla 2, el valor del índice de madurez dlde implica inicialmente cuando empieza su crecimiento que los frutos tienen forma oblonga en las tres fincas y, que a medida que se acerca la maduración o estadio 8 de la escala $\mathrm{BBCH}$ para su cosecha como lo describió para los frutales caducifolios (Meier, 1997; 2009), adquieren la forma redondeada típica del fruto de duraznero; cerca de la época de maduración, el valor de la relación entre los dos diámetros tiende a la unidad tal como lo reportaron Casierra-Posada et al. (2004) e indicaría que se puede cosechar primero en Pamplonita debido a que alcanza el valor de la unidad más temprano que las otras dos altitudes o grupos y que esta se realizaría de último en Chitagá.

La tabla 2 muestra también que es mayor el peso seco en Chitagá probablemente atribuible al rápido incremento del peso seco en la última fase del crecimiento de los frutos (Casierra-Posada et al., 2004). La temperatura, la variación de la luz en función de la latitud y el régimen pluviométrico a lo largo del año son los principales factores que afectan la fenología del duraznero, cultivo que presenta interrupción del crecimiento o crecimiento rítmico, es decir, alterna flujos de actividad y reposo (Sanabria, 2011; Pinzón et al., 2014). 


\section{CONCLUSIONES}

Del análisis discriminante se evidenció una separación del piso térmico del sector de Chitagá con Pamplonita, teniendo un área común con Pamplona, lo que implicó más similaridad entre Pamplona y Pamplonita y Chitagá y Pamplona, siendo los más diferenciados las fincas de duraznero Pamplonita y Chitagá, seguramente por el hecho de encontrarse en cuencas diferentes.

Las dos funciones discriminantes obtenidas generaron una proporción de traza de $89,3 \%$, lo cual representó una alta separación porcentual entre funciones, lo que significó que prácticamente una sola función pudiera usarse para efectos de la separación de las fincas analizadas.

Los promedios del producto de largo y ancho de limbos, área digital de los limbos, cociente del diámetro longitudinal y ecuatorial de frutos así como el peso seco de los frutos fueron más extremos en las fincas de Chitagá y Pamplonita, siendo Chitagá la de máximos valores promedios en las variables morfométricas y Pamplonita se llevó el máximo promedio de peso de frutos.

\section{REFERENCIAS BIBLIOGRÁFICAS}

Arcila, P. y C.B. Chaves. 1995. Desarrollo foliar del cafeto en tres densidades de siembra. Cenicafé 46(1), 5-20.

Aribal, L.G., J.G. Bonggay y E.S. Fernando. 2017. Leaf size indices and structure of the peat swamp forest. Global J. Environ. Sci. Manag. 3(1), 63-74. Doi: 10.22034/ gjesm.2017.03.01.007

Avilán, L., E. Soto, M. Pérez, C. Marín, M. Rodríguez y J. Ruiz. 2009. Comportamiento fenológico de la raza antillana de aguacate en la región centro-norte costera de Venezuela. Agron. Trop. 59(1), 5-14.

Beckman, R. y R. Cook. 1983. Outlier ... s (with discussion). Technometries 25(29), 119-149.

Blanco, J.A., O. Balocchi e I. López. 2010. Variabilidad fenotípica en accesiones de Bromus valdivianus Phil. Agro Sur 38(2), 68-79. Doi: 10.4206/agrosur.2010.v38n2-02

Campos, T. de J. 2013. Especies y variedades de hoja caduca en Colombia. pp. 47-64. En: Miranda, D., G. Fischer y C. Carranza (eds.). Los frutales caducifolios en Colombia - Situación actual, sistemas de cultivo y plan de desarrollo. Sociedad Colombiana de Ciencias Hortícolas, Bogotá.

Cárdenas, J. y G. Fischer. 2013. Clasificación botánica y morfología de manzano, peral, duraznero y ciruelo. pp. 21-30. En: Miranda, D., G. Fischer y C. Carranza (eds.). Los frutales caducifolios en Colombia - Situación actual, sistemas de cultivo y plan de desarrollo. Sociedad Colombiana de Ciencias Hortícolas, Bogotá.

Carranza, C. y D. Miranda. 2013. Zonificación actual de los sistemas de producción de frutales caducifolios en Colombia. pp. 67-86. En: Miranda, D., G. Fischer y C. Carranza (eds.). Los frutales caducifolios en Colombia - Situación actual, sistemas de cultivo y plan de desarrollo. Sociedad Colombiana de Ciencias Hortícolas, Bogotá.

Casierra-Posada, F,. V.E. Barreto y O.L. Fonseca. 2004. Crecimiento de frutos y ramas de duraznero (P. persica $\mathrm{L}$. Batsch, cv. 'Conservero') en los altiplanos colombianos. Agron. Colomb. 22(1), 40-45.

Delgado, I., H. Jorge, H. García, N. Bernal, F.R. Díaz, I. Jorge, A. Bernal, O. Rivera, P. Machado, F. Barroso, M. Buedo, S. Reyes, A. Gallardo, J. Barroso, J. Rosa, L.F. Machado, J.L. Pérez y J.R. Fardales. 2008. Utilización de los análisis multivariados en la clasificación de ambientes en caña de azúcar. Centro Agríc. 35(4), 53-57.

Fernández, G. 2013. Caracterización de los productores de durazno (P. persica L. Batsch) del municipio de Cácota, Norte de Santander. Trabajo de grado. Universidad de Pamplona, Pamplona, Colombia.

Fischer, G. 2013. Comportamiento de los frutales caducifolios en el trópico. pp. 31-46. En: Miranda, D., G. Fischer y C. Carranza (eds.). Los frutales caducifolios en Colombia - Situación actual, sistemas de cultivo y plan de desarrollo. Sociedad Colombiana de Ciencias Hortícolas, Bogotá.

Fischer, G. 1995. Effect of root-zone temperatura and tropical altitude on the growth, development and fruit quality of cape gooseberry (Physalis peruviana L.). Tesis de doctorado. Humboldt-Universität zu Berlin, Berlín, Alemania.

Fischer, G., F. Casierra-Posada y C. Villamizar. 2010. Producción forzada de duraznero (P. persica (L.) Batsch) en el altiplano tropical de Boyacá (Colombia). Rev. Colomb. Cienc. Hortíc. 4(1), 19-32. Doi: 10.17584/ rcch.2010v4i1.1223

Fischer, G., F. Ramírez y F. Casierra-Posada. 2016. Ecophysiological aspects of fruit crops in the era of climate change. A review. Agron. Colomb. 34(2), 190-199. Doi: 10.15446/agron.colomb.v34n2.56799

Garrido, E.M., T.G. Rujano, A. Torres, E. Sangronis, J.A. Martínez, L.C. Chaparro y L. Sánchez. 2013. Análisis de las características físicas y químicas del fruto de mango (Mangifera indica L.) 'Bocado' de tres localidades del Estado Cojedes, Venezuela. Rev. Venez. Cienc. Tecnol. Aliment. 4(2), 189-206.

Gómez, D., O. Solano, V. Osorio, L. Huamán, G. Ramón, J.I. Condado, M. Gallardo y Y.M. Quinteros. 2013. Identificación de observaciones influyentes en la discriminación de muestras del género Minthostachys de Cajatambo y Unchos. Pesquimat 15(2), 15-28. 
Gordon-Colón, E., L.M. Suárez-Villasmil, L. Polanco y E.M. Barreto-Pittol. 2013. Distribución geográfica y variación foliar de Montrichardia arborescens en Venezuela. Acta Biol. Venez. 33(1-2), 23-42.

Henderson, A. 2006. Traditional morphometrics in plant systematics and its role in palm systematics. Bot. J. Linn. Soc. 151(1), 103-111. Doi: 10.1111/j.1095-8339.2006.00526.x

Jobson, J.D. 1992. Applied multivariate data analysis. Vol. II: Categorical and multivariate methods. Springer Science and Business Media, New York, NY. Doi: 10.1007/978-1-4612-0921-8

MADR. 2012. Anuario estadístico de fruta y hortalizas 2007-2011 y sus calendarios de siembras y cosechas. Resultados evaluaciones agropecuarias municipales 2011. Ministerio de Agricultura y Desarrollo Rural de Colombia, Bogotá.

Manly, B.F.J. y A.J.A. Navarro. 2017. Multivariate statistical methods. A primer. $3^{\text {rd }}$ ed. Chapman \& Hall, CRC Press, Boca Raton, FL.

Meier U. 1997. BBCH-Monograph. Growth stages of plants. Blackwell Wissenschaftsverlag, Berlin, Alemania.

Meier U., H. Bleiholder, L. Buhr, C. Feller, H. Hack, M. Heß, P.D. Lancashire, U. Schnock, R. Stauß, T. van den Boom, E. Weber y P. Zwerger. 2009. The BBCH system to coding the phenological growth stages of plants-history and publications. J. Cultiv. Plants 61, 41-52.

Minasny, B., B.P. Malone y A.B. McBratney. 2012. digital soil assessment and beyond. CRC Press, Taylor \& Francis Group, London, UK.

Oppong, F.B. and S.Y. Agbedra. 2016. Assessing univariate and multivariate normality. a guide for non-statisticians. Math. Theory Modeling 6(2), 26-33.

Pintaluba, N. and P.A. Luaces. 2013. Caracterización de frutas comestibles de especies nativas de uso popular en el parque provincial "Salto encantado del valle del Cuñá-Pirú - misiones". Bonplandia 22(2), 191-201.

Pinzón, E.H., A.C. Morillo y G. Fischer. 2014. Aspectos fisiológicos del duraznero (P. persica [L.] Batsch.) en el trópico alto. Una revisión. Rev. UDCA Act. Div. Cient. $17(2), 401-411$

Rasband, W. S. 2010. ImageJ. U.S. National Institute of Health, Bethesda, MD, USA.

Sanabria, A. 2011. Crecimiento vegetativo, reproductivo y fenología. pp. 21-27. En: Aular, J., M. Casares y J. Gebaüer (eds.). Manejo hortícola de huertos de duraznero. Universidad Centro Occidental "Lisandro Alvarado", Barquisimeto, Venezuela.

William, B.K. 1983. Some observations of the use of discriminant analysis in ecology. Ecol. 64(5), 1283-1291. Doi: 10.2307/1937836 